\title{
Closed-form calculation of infinite products of Glaisher-type related to Dirichlet series
}

\author{
Matthew Perkins' ${ }^{1}$ - Robert A. Van $\operatorname{Gorder}^{1}$
}

Received: 13 October 2017 / Accepted: 7 May 2018 / Published online: 31 August 2018

(C) The Author(s) 2018

\begin{abstract}
Building on recent work involving the computation of generalizations of Glaisher-type products over the primes by differentiation of the Euler product identity, in the present paper we generalize this approach in order to obtain closed-form expressions of more general infinite products which correspond to Dirichlet series. In this way, we obtain an elegant method to compute a variety of interesting infinite products, and some infinite double products. The Bendersky-Adamchik constants enter into a number of our results, and appear quite fundamental to these infinite products. A number of concrete examples are given in order to illustrate the general principle, including cases where these powers involve the divisor function or the Möbius function. We also consider general families of infinite products over the prime numbers (rather than the natural numbers) in order to obtain other new infinite product identities. Infinite products over terms directly involving Bendersky-Adamchik constants are considered, and these are helpful for later extending our approach to infinite double products over both the lattice of natural numbers and the lattice of prime numbers.
\end{abstract}

Keywords Infinite products - Glaisher products - Bendersky-Adamchik constants · Infinite double products

Mathematics Subject Classification Primary 40A20 · Secondary 11M06

$凶$ Robert A. Van Gorder

Robert.VanGorder@maths.ox.ac.uk

1 Mathematical Institute, University of Oxford, Andrew Wiles Building, Radcliffe Observatory Quarter, Woodstock Road, Oxford OX2 6GG, UK 


\section{Introduction}

The Bendersky-Adamchik constants $D_{m}$ (see [2-5]) have seen use in a variety of areas related to infinite series and products, as well as to definite integrals. These constants originally appeared in [4], where $\log \left(D_{m}\right)$ arises as the constant term in the EulerMaclaurin summation of $\log \left(1^{1^{m}} 2^{2^{m}} 3^{3^{m}} \ldots x^{x^{m}}\right)$. The constants were formalized by way of the limit

$$
\log D_{m}=\lim _{n \rightarrow \infty}\left(\sum_{k=1}^{n} k^{m} \log k-p(n, m)\right),
$$

where the function $p(n, m)$ is given by

$$
\begin{aligned}
p(n, m)= & \frac{n^{m}}{2} \log n+\frac{n^{m+1}}{m+1}\left(\log n-\frac{1}{m+1}\right) \\
& +m ! \sum_{j=1}^{m} \frac{n^{m-j} B_{j+1}}{(j+1) !(m-j) !}\left(\log n+\left(1-\delta_{m j}\right) \sum_{l=1}^{j} \frac{1}{m-l+1}\right),
\end{aligned}
$$

with $B_{j}$ the $j$ th Bernoulli number. The first few Bendersky-Adamchik constants are therefore given by

$$
\begin{aligned}
& \log D_{1}=\lim _{n \rightarrow \infty}\left(\sum_{k=1}^{n} k \log k-\left(\frac{n^{2}}{2}+\frac{n}{2}+\frac{1}{12}\right) \log n+\frac{n^{2}}{4}\right) \\
& \log D_{2}=\lim _{n \rightarrow \infty}\left(\sum_{k=1}^{n} k^{2} \log k-\left(\frac{n^{3}}{3}+\frac{n^{2}}{2}+\frac{n}{6}\right) \log n+\frac{n^{3}}{9}-\frac{n}{12}\right), \\
& \log D_{3}=\lim _{n \rightarrow \infty}\left(\sum_{k=1}^{n} k^{3} \log k-\left(\frac{n^{4}}{4}+\frac{n^{3}}{2}+\frac{n^{2}}{4}-\frac{1}{120}\right) \log n+\frac{n^{4}}{16}-\frac{n^{2}}{12}\right) .
\end{aligned}
$$

Exponentiation of (1.1) displays how these constants emulate Stirling's formula; as $D_{0}=\sqrt{2 \pi}$ and $\prod_{k=1}^{n} k=n !$, one obtains the identities

$$
\begin{aligned}
& D_{0}=\lim _{n \rightarrow \infty}\left(\frac{\prod_{k=1}^{n} k}{e^{-n} n^{n+\frac{1}{2}}}\right), \\
& D_{1}=\lim _{n \rightarrow \infty}\left(\frac{\prod_{k=1}^{n} k^{k}}{e^{-\frac{n^{2}}{4}} n^{\frac{n^{2}}{2}}+\frac{n}{2}+\frac{1}{12}}\right) \text {, } \\
& D_{2}=\lim _{n \rightarrow \infty}\left(\frac{\prod_{k=1}^{n} k^{k^{2}}}{e^{-\frac{n^{3}}{9}+\frac{n}{12} n^{\frac{n^{3}}{3}}+\frac{n^{2}}{2}+\frac{n}{6}}}\right) \text {, }
\end{aligned}
$$

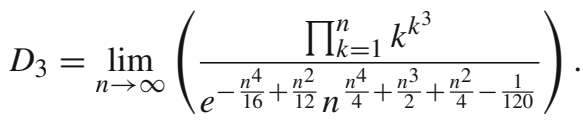


The Bendersky-Adamchik constants $D_{m}$ arise in the study of the multiple gamma function $\Gamma_{n}(z)$ [3], which generalizes the gamma function, the polygamma function $\psi_{n}(z)$ [2], and appears in expressions related to the derivative of the Riemann and Hurwitz zeta functions [2]. Note that $D_{1}$ is equal to $A$, the Glaisher-Kinkelin constant, which has historically seen more attention $[10,11,26]$, appearing in a number of product, series, and integral relations, including the formula

$$
\int_{0}^{\infty} \frac{x \log x}{e^{2 \pi x}-1} \mathrm{~d} x=\frac{1}{24}-\frac{1}{2} \log D_{1}
$$

as well as the famous product identities which motivated work in [24]; see also [13]. The next couple of Bendersky-Adamchik constants have also been of interest, and have been used to find closed-form representations for series [6] such as

$$
\sum_{n=1}^{\infty} \frac{\zeta(2 n)}{(n+1) 4^{n}}=\frac{7}{6}-\log 2+14 \log D_{2},
$$

for integral formulas [4] such as

$$
\begin{aligned}
& \int_{0}^{1}\left(\frac{(1-x)^{2}-\log ^{2} x}{x \log ^{3} x}-1\right) \frac{\mathrm{d} x}{(1+x) \log x} \\
& \quad=\frac{1}{2} \log \pi-\frac{11}{36}-\frac{7}{90} \log 2-3 \log D_{1}+10 \log D_{3}
\end{aligned}
$$

as well as numerous multiple integral formulas, such as

$$
\int_{0}^{\frac{1}{2}} \int_{0}^{x} \log (\sin (\pi t)) \mathrm{d} t \mathrm{~d} x=-\frac{1}{8} \log 2-\frac{7}{4} \log D_{2} .
$$

Particular infinite products over the natural numbers and the primes have been calculated (see [24]) via the equation

$$
\prod_{n=1}^{\infty} n^{1 / n^{x}}=\left(\prod_{n=1}^{\infty} p_{n}^{1 /\left(p_{n}^{x}-1\right)}\right)^{\zeta(x)}
$$

where $p_{n}$ represents the $n$th prime number. This equation was derived through differentiation of the Euler product form of the Riemann zeta function [24]. Additionally, for natural numbers $m$, the closed-form product identity

$$
\prod_{n=1}^{\infty} p_{n}^{1 /\left(p_{n}^{2 m}-1\right)}=\frac{\left(D_{2 m-1}\right)^{\frac{2 m}{B_{2 m}}}}{2 \pi e^{\gamma}}
$$

was found in [24], utilizing the Bendersky-Adamchik constants $D_{2 m-1}$ (see [2-5]). 
In the present paper we make use of the Bendersky-Adamchik constants in order to obtain closed-form evaluations of more general infinite products of the form

$$
\prod_{n=1}^{\infty} n^{a_{n} / n^{x}}
$$

where the $a_{n}$ arise as coefficients in certain Dirichlet series. In Sect. 2 we generalize (1.2) in order to account for products in such a form. A number of concrete examples are given, such as for when $a_{n}$ involves the divisor function or the Möbius function. In Sect. 3 we consider general families of infinite products over prime numbers (rather than natural numbers) in order to obtain other new infinite product identities. In Sect. 4 we turn our attention to infinite products of functions of Bendersky-Adamchik constants, obtaining, for example,

$$
\prod_{m=1}^{\infty} D_{2 m}^{\frac{(-1)^{m+1} \pi^{2 m}}{(2 m) !}}=\frac{2}{\sqrt{e}}
$$

which is Corollary 4.3. In Sect. 5, we consider similar approaches for infinite double products.

\section{Infinite products related to certain Dirichlet series}

In [24], term-wise differentiation of the famous Euler product

$$
\zeta(x)=\prod_{n=1}^{\infty}\left(1-p_{n}^{-x}\right)^{-1}
$$

results in the derivative

$$
\zeta^{\prime}(x)=-\zeta(x) \sum_{n=1}^{\infty} \frac{\log p_{n}}{p_{n}^{x}-1},
$$

which is valid for $x>1$; this was used to obtain a variety of infinite product identities of Glaisher type. This relation directly leads to (1.2). In the following, we will repeatedly apply (2.1) to derive new infinite product relations. To do so, we will utilize Dirichlet series intimately related with the zeta function. It is well known that many relations for generalized zeta functions exist, and we shall consider the more general form

$$
g(x)=\sum_{n=1}^{\infty} \frac{a_{n}(k)}{n^{x}}=h(x) \zeta^{k}(x),
$$

which was discussed in [12]. We will consider cases where all the $a_{n}(k)$ are real, the sum defining $g(x)$ converges uniformly, and $g(x)$ is differentiable on $x>1$. We shall 
suppress the $k$, writing $a_{n}(k)=a_{n}$ for convenience, unless $k$ is otherwise needed for clarity. Differentiating (2.2), and using (2.1), we have

$$
g^{\prime}(x)=\sum_{n=1}^{\infty} \frac{-a_{n} \log n}{n^{x}}=\zeta^{k}(x)\left(h^{\prime}(x)-k h(x) \sum_{n=1}^{\infty} \frac{\log p_{n}}{p_{n}^{x}-1}\right) .
$$

Rearranging terms and applying the exponential function to both sides of (2.3) yields the following.

Theorem 2.1 We have that

$$
\prod_{n=1}^{\infty} n^{a_{n} / n^{x}}=\left(e^{-h^{\prime}(x)}\left(\prod_{n=1}^{\infty} p_{n}^{\frac{1}{p_{n}^{x}-1}}\right)^{k h(x)}\right)^{\zeta^{k}(x)} .
$$

Before moving on we shall ensure that the representation is indeed uniformly convergent, since we require uniform convergence to justify term-wise differentiation. However, as the series we will consider are of the form

$$
\sum_{n=1}^{\infty} \frac{a_{n}}{n^{x}}=f(\zeta(x))
$$

we can exploit the fact that the series converge on $x>1$ to use the Weierstrass $M$-Test. For any $\varepsilon>0$, and $x \in[1+\varepsilon, \infty)$, we have

$$
\left|\frac{a_{n}}{n^{x}}\right| \leq\left|\frac{a_{n}}{n^{1+\varepsilon}}\right|
$$

Once we set $M_{n}=\left|\frac{a_{n}}{n^{1+\varepsilon}}\right|$,

$$
\sum_{n=1}^{\infty} M_{n}=f(\zeta(1+\varepsilon))<\infty
$$

via the Weierstrass $M$-Test it follows that for all $\varepsilon>0$ the series converges uniformly on $[1+\varepsilon, \infty)$, and hence on $(1, \infty)$.

We now refer to [12,22] for further Dirichlet series and utilize them to form a collection of results below, motivated by the general relation given in Theorem 2.1. The region of validity in our analytic results is $x>1$, unless otherwise stated.

2.1 Case 1: $a_{n}=(-1)^{n+1}$

Consider the Dirichlet $\eta$ function

$$
\eta(x)=1-\frac{1}{2^{x}}+\frac{1}{3^{x}}-\frac{1}{4^{x}}+\frac{1}{5^{x}}-\cdots=\sum_{n=1}^{\infty} \frac{(-1)^{n+1}}{n^{x}} .
$$


It is an easy deduction (see $[18,25])$ that

$$
\eta(x)=\left(1-2^{1-x}\right) \zeta(x)
$$

and hence we take $h(x)=1-2^{1-x}$, which has derivative $h^{\prime}(x)=2^{1-x} \log 2$. As $e^{-h^{\prime}(x)}=e^{-2^{1-x} \log 2}=2^{-2^{1-x}}$, employing $k=1$ in Theorem 2.1 yields

$$
\prod_{n=1}^{\infty} n^{(-1)^{n+1} / n^{x}}=\left(2^{-2^{1-x}} \prod_{n=1}^{\infty} p_{n}^{\frac{1-2^{1-x}}{p_{n}^{x}-1}}\right)^{\zeta(x)}
$$

Wishing to substitute a value of $x$ that gives a closed-form for the prime product, we take the simplest case $x=2$, which gives the following corollary to Theorem 2.1.

Corollary 2.1 We have the identity

$$
\prod_{n=1}^{\infty} n^{(-1)^{n+1} / n^{2}}=\left(\frac{D_{1}^{12}}{4 \pi e^{\gamma}}\right)^{\pi^{2} / 12} .
$$

\subsection{Case 2: $a_{n}=d(n)$, the divisor function}

Note that

$$
\zeta^{2}(x)=\sum_{n=1}^{\infty} \frac{d(n)}{n^{x}}
$$

where $d(n)$ denotes the divisor function, i.e., the number of divisors of an integer $n$, including 1 and itself [14]. The divisor function obeys the well-known asymptotic relation (see [12])

$$
\frac{1}{n} \sum_{k=1}^{n} d(k) \sim \log n+2 \gamma-1
$$

where $\gamma$ is the Euler-Mascheroni constant. Applying Theorem 2.1 with $k=2$ and $h(x)=1$, we have

$$
\prod_{n=1}^{\infty} n^{d(n) / n^{x}}=\left(\prod_{n=1}^{\infty} p_{n}^{1 /\left(p_{n}^{x}-1\right)}\right)^{2 \zeta^{2}(x)}
$$

Taking $x=2$, we obtain the following identity.

Corollary 2.2 We have

$$
\prod_{n=1}^{\infty} n^{d(n) / n^{2}}=\left(\frac{D_{1}^{12}}{2 \pi e^{\gamma}}\right)^{\pi^{4} / 18}
$$


Furthermore, there is a well-known Dirichlet series relating $d\left(n^{2}\right)$ to the zeta function, that we will use:

$$
\frac{\zeta^{3}(x)}{\zeta(2 x)}=\sum_{n=1}^{\infty} \frac{d\left(n^{2}\right)}{n^{x}}
$$

Taking $k=3$ and $h(x)=\zeta(2 x)^{-1}$ in Theorem 2.1 , and noting

$$
h^{\prime}(x)=2 h(x)\left(\sum_{n=1}^{\infty} \frac{\log p_{n}}{p_{n}^{2 x}-1}\right),
$$

we have

$$
\prod_{n=1}^{\infty} n^{d\left(n^{2}\right) / n^{x}}=\left(\frac{\left(\prod_{n=1}^{\infty} p_{n}^{1 /\left(p_{n}^{x}-1\right)}\right)^{3}}{\left(\prod_{n=1}^{\infty} p_{n}^{1 /\left(p_{n}^{2 x}-1\right)}\right)^{2}}\right)^{\frac{\zeta^{3}(x)}{\zeta(2 x)}}
$$

For example, taking $x=2$, we have the following.

Corollary 2.3 We have

$$
\prod_{n=1}^{\infty} n^{d\left(n^{2}\right) / n^{2}}=\left(\frac{D_{1}^{36} D_{3}^{240}}{2 \pi e^{\gamma}}\right)^{5 \pi^{2} / 12}
$$

There is also another Dirichlet series relating the divisor function and squares, giving the relation

$$
\frac{\zeta^{4}(x)}{\zeta(2 x)}=\sum_{n=1}^{\infty} \frac{d^{2}(n)}{n^{x}}
$$

Taking $k=4$ and $h(x)=(\zeta(2 x))^{-1}$ in Theorem 2.1, we obtain the product relation

$$
\prod_{n=1}^{\infty} n^{d^{2}(n) / n^{x}}=\left(\frac{\left(\prod_{n=1}^{\infty} p_{n}^{1 /\left(p_{n}^{x}-1\right)}\right)^{2}}{\left(\prod_{n=1}^{\infty} p_{n}^{1 /\left(p_{n}^{2 x}-1\right)}\right)}\right)^{\frac{2 \zeta^{4}(x)}{\zeta(2 x)}}
$$

Setting $x=2$, we recover the following.

Corollary 2.4 We have

$$
\prod_{n=1}^{\infty} n^{(d(n) / n)^{2}}=\left(\frac{D_{1}^{24} D_{3}^{120}}{2 \pi e^{\gamma}}\right)^{5 \pi^{4} / 36}
$$




\subsection{Case 3: $a_{n}=d_{m}(n)$}

Let the function $d_{m}(n)$ represent the number of ways the integer $n$ can be represented as a product of $m$ factors (including unity, up to $m-1$ times). Hence $d_{m}(n)$ generalizes the divisor function, with $m=2$ giving $d_{2}(n)=d(n)$. It is well-known (see [12]) that

$$
\sum_{n=1}^{\infty} \frac{d_{m}(n)}{n^{x}}=\zeta^{m}(x)
$$

and applying Theorem 2.1 with $k=m$ and $h(x)=1$, we have

$$
\prod_{n=1}^{\infty} n^{d_{m}(n) / n^{x}}=\left(\prod_{n=1}^{\infty} p_{n}^{1 /\left(p_{n}^{x}-1\right)}\right)^{m \zeta^{m}(x)} .
$$

Taking $x=2$, this gives the following identity.

Corollary 2.5 We have

$$
\prod_{n=1}^{\infty} n^{d_{m}(n) / n^{2}}=\left(\frac{D-1^{12}}{2 \pi e^{\gamma}}\right)^{\frac{m \pi^{2 m}}{6^{m}}} .
$$

\subsection{Case 4: $a_{n}=\sigma_{m}(n)$, the sum of $m$ th powers of divisors of $n$}

The standard divisor function can be represented as $d(n)=\sum_{d \mid n}$. The sum of the $k$ th powers of divisors of $n$, defined formally as

$$
\sigma_{m}(n)=\sum_{d \mid n} d^{m},
$$

gives another generalization of the divisor function, with the $m=0$ case giving $\sigma_{0}(n)=d(n)$. Note that this generalization does not require $m$ to be an integer. Like $\sigma_{0}(n)=d(n), \sigma_{1}(n)$ satisfies several asymptotic expressions, notably (see [12])

$$
\frac{1}{n^{2}} \sum_{k=1}^{n} \sigma_{1}(k) \sim \frac{\pi^{2}}{12},
$$

which is similar to (2.4). As with $d_{m}(n)$, there is a Dirichlet series relating $\sigma_{m}(n)$ to $\zeta$,

$$
\sum_{n=1}^{\infty} \frac{\sigma_{m}(n)}{n^{x}}=\zeta(x) \zeta(x-m),
$$


which holds provided that $x>\max \{m+1,1\}$. Applying Theorem 2.1 with $h(x)=$ $\zeta(x-m)$, and noting that $h^{\prime}(x)$ can be computed with (2.1), we have

$$
\prod_{n=1}^{\infty} n^{\sigma_{m}(n) / n^{x}}=\left(\prod_{n=1}^{\infty} p_{n}^{1 /\left(p_{n}^{x}-1\right)} \prod_{n=1}^{\infty} p_{n}^{1 /\left(p_{n}^{x-m}-1\right)}\right)^{\zeta(x) \zeta(x-m)}
$$

Taking $x=2 t, x-m=2 s$, for $s, t \in \mathbb{N}$ we have

$$
\prod_{n=1}^{\infty} n^{\sigma_{2(t-s)}(n) / n^{2 t}}=\left(\frac{\left(D_{2 t-1}\right)^{\frac{t}{B_{2 t}}}\left(D_{2 s-1}\right)^{\frac{s}{B_{2 s}}}}{2 \pi e^{\gamma}}\right)^{2 \zeta(2 t) \zeta(2 s)}
$$

which simplifies to the following form.

Corollary 2.6 We have

$$
\prod_{n=1}^{\infty} n^{\sigma_{2}(n) / n^{4}}=\left(\frac{D_{1}}{2 \pi e^{\gamma} D_{3}^{10}}\right)^{\pi^{6} / 45}
$$

\subsection{Case 5: $a_{n}=\lambda(n)$, Liouville's function}

Liouville's function is defined as $\lambda(n)=(-1)^{\Omega(n)}$, where $\Omega(n)$ represents the number of prime divisors of $n$ including multiplicity [16]. Liouville's function is completely multiplicative, as it is easily seen that $\Omega(1)=0$ and $\Omega(n m)=\Omega(n)+\Omega(m)$. This fact is useful in the derivation of the classical relation (see [16])

$$
\sum_{n=1}^{\infty} \frac{\lambda(n)}{n^{x}}=\frac{\zeta(2 x)}{\zeta(x)}
$$

Using $k=-1$ and $h(x)=\zeta(2 x)$ in Theorem 2.1, we find

$$
\prod_{n=1}^{\infty} n^{\lambda(n) / n^{x}}=\left(\frac{\left(\prod_{n=1}^{\infty} p_{n}^{1 /\left(p_{n}^{2 x}-1\right)}\right)^{2}}{\prod_{n=1}^{\infty} p_{n}^{1 /\left(p_{n}^{x}-1\right)}}\right)^{\frac{\zeta(2 x)}{\zeta(x)}},
$$

which for $x=2$ gives the following expression.

Corollary 2.7 We have

$$
\prod_{n=1}^{\infty} n^{\lambda(n) / n^{2}}=\left(\frac{1}{2 \pi e^{\gamma} D_{1}^{12} D_{3}^{240}}\right)^{\pi^{2} / 15}
$$


In [12], there are several Dirichlet series relations containing both the divisor and Liouville functions, including

$$
\begin{aligned}
& \sum_{n=1}^{\infty} \frac{\lambda(n) d\left(n^{2}\right)}{n^{x}}=\frac{\zeta^{2}(2 x)}{\zeta^{3}(x)}, \\
& \sum_{n=1}^{\infty} \frac{\lambda(n) d^{2}(n)}{n^{x}}=\frac{\zeta^{3}(2 x)}{\zeta^{4}(x)}
\end{aligned}
$$

From these equations, we derive the infinite product relations

$$
\prod_{n=1}^{\infty} n^{\lambda(n) d\left(n^{2}\right) / n^{x}}=\left(\frac{\left(\prod_{n=1}^{\infty} p_{n}^{1 /\left(p_{n}^{2 x}-1\right)}\right)^{4}}{\left(\prod_{n=1}^{\infty} p_{n}^{1 /\left(p_{n}^{x}-1\right)}\right)^{3}}\right)^{\frac{\zeta^{2}(2 x)}{\zeta^{3}(x)}}
$$

and

$$
\prod_{n=1}^{\infty} n^{\lambda(n) d^{2}(n) / n^{x}}=\left(\frac{\left(\prod_{n=1}^{\infty} p_{n}^{1 /\left(p_{n}^{2 x}-1\right)}\right)^{3}}{\left(\prod_{n=1}^{\infty} p_{n}^{1 /\left(p_{n}^{x}-1\right)}\right)^{2}}\right)^{\frac{2 \zeta^{3}(2 x)}{\zeta^{4}(x)}}
$$

respectively. Taking $x=2$, we obtain, respectively, the following formulas.

Corollary 2.8 We have

$$
\prod_{n=1}^{\infty} n^{\lambda(n) d\left(n^{2}\right) / n^{2}}=\left(\frac{1}{2 \pi e^{\gamma} D_{1}^{36} D_{3}^{480}}\right)^{2 \pi^{2} / 75}
$$

and

$$
\prod_{n=1}^{\infty} n^{\lambda(n) d^{2}(n) / n^{2}}=\left(\frac{1}{2 \pi e^{\gamma} D_{1}^{24} D_{3}^{360}}\right)^{4 \pi^{4} / 1125}
$$

\subsection{Case 6: $a_{n}=\mu(n)$, the Möbius function}

The Möbius function, $\mu(n)$, takes the value zero for integers $n$ with any prime factor repeated, and otherwise takes the value $(-1)^{m}$, where $m$ is the number of prime factors of $n$. For our interests, there are two relevant Dirichlet series relations for the Möbius 
function (see [21]):

$$
\begin{aligned}
\sum_{n=1}^{\infty} \frac{\mu(n)}{n^{x}} & =\frac{1}{\zeta(x)}, \\
\sum_{n=1}^{\infty} \frac{\mu^{2}(n)}{n^{x}} & =\frac{\zeta(x)}{\zeta(2 x)} .
\end{aligned}
$$

Taking $k=-1$ with $h(x)=1$, and separately $k=1$ with $h(x)=\zeta(2 x)^{-1}$, we apply Theorem 2.1 to find

$$
\begin{aligned}
& \prod_{n=1}^{\infty} n^{\mu(n) / n^{x}}=\left(\prod_{n=1}^{\infty} p_{n}^{1 /\left(p_{n}^{x}-1\right)}\right)^{-\frac{1}{\zeta(x)}}, \\
& \prod_{n=1}^{\infty} n^{\mu^{2}(n) / n^{x}}=\left(\frac{\prod_{n=1}^{\infty} p_{n}^{1 /\left(p_{n}^{x}-1\right)}}{\left(\prod_{n=1}^{\infty} p_{n}^{1 /\left(p_{n}^{2 x}-1\right)}\right)^{2}}\right)^{\frac{\zeta(x)}{\zeta(2 x)}},
\end{aligned}
$$

respectively. Considering the special case $x=2$, we obtain the following product relations.

Corollary 2.9 We have

$$
\prod_{n=1}^{\infty} n^{\mu(n) / n^{2}}=\left(\frac{2 \pi e^{\gamma}}{D_{1}^{12}}\right)^{6 / \pi^{2}}
$$

and

$$
\prod_{n=1}^{\infty} n^{(\mu(n) / n)^{2}}=\left(2 \pi e^{\gamma} D_{1}^{12} D_{3}^{240}\right)^{15 / \pi^{2}} .
$$

Note that while (2.6) holds for $x>1, \frac{1}{\zeta(x)}$ is not defined at $x=1$ without analytic continuation. However, it is also known that [1]

$$
\sum_{n=1}^{\infty} \frac{\mu(n) \log n}{n}=-1
$$

which results in a corresponding product relation for $x=1$, namely the following identity.

Proposition 2.1 We have

$$
\prod_{n=1}^{\infty} n^{\mu(n) / n}=\frac{1}{e}
$$




\subsection{Case 7: $a_{n}=\theta(n)$}

The function $\theta(n)$ is defined as the number of ordered pairs $(a, b)$, with $\operatorname{gcd}(a, b)=1$, such that $n=a b[12]$. It is known that $\theta(n)$ satisfies the equation

$$
\frac{\zeta^{2}(x)}{\zeta(2 x)}=\sum_{n=1}^{\infty} \frac{\theta(n)}{n^{x}}
$$

Taking $k=2$ and $h(x)=\zeta(2 x)^{-1}$ in Theorem 2.1, we have

$$
\prod_{n=1}^{\infty} n^{\theta(n) / n^{x}}=\left(\frac{\prod_{n=1}^{\infty} p_{n}^{1 /\left(p_{n}^{x}-1\right)}}{\prod_{n=1}^{\infty} p_{n}^{1 /\left(p_{n}^{2 x}-1\right)}}\right)^{\frac{2 \zeta^{2}(x)}{\zeta(2 x)}}
$$

and on setting $x=2$, we find the following special case.

Corollary 2.10 We have

$$
\prod_{n=1}^{\infty} n^{\theta(n) / n^{2}}=\left(D_{1} D_{3}^{10}\right)^{60}
$$

Just as with the divisor function, $\theta(n)$ appears in another Dirichlet series multiplied by the Liouville function [12], namely

$$
\sum_{n=1}^{\infty} \frac{\lambda(n) \theta(n)}{n^{x}}=\frac{\zeta(2 x)}{\zeta^{2}(x)}
$$

Taking $k=-2$ and $h(x)=\zeta(2 x)$, we find

$$
\prod_{n=1}^{\infty} n^{\lambda(n) \theta(n) / n^{x}}=\left(\frac{\prod_{n=1}^{\infty} p_{n}^{1 /\left(p_{n}^{2 x}-1\right)}}{\prod_{n=1}^{\infty} p_{n}^{1 /\left(p_{n}^{x}-1\right)}}\right)^{\frac{2 \zeta(2 x)}{\zeta^{2}(x)}}
$$

and upon setting $x=2$, we have the following special case.

Corollary 2.11 We have

$$
\prod_{n=1}^{\infty} n^{\lambda(n) \theta(n) / n^{2}}=\left(\frac{1}{D_{1} D_{3}^{10}}\right)^{48 / 5}
$$




\section{Infinite products over the prime numbers}

The general relation Theorem 2.1 can also be used to give families of infinite products defined over the prime numbers. As an example, noting that

$$
\frac{1}{p_{n}^{2 m}+1}=\frac{1}{p_{n}^{2 m}-1}-\frac{2}{p_{n}^{4 m}-1}
$$

we can substitute $x=2 m$ and $x=4 m$ into Theorem 2.1 , in order to calculate the following family of products.

Corollary 3.1 We have

$$
\prod_{n=1}^{\infty} p_{n}^{1 /\left(p_{n}^{2 m}+1\right)}=\left(\frac{2 \pi e^{\gamma}\left(D_{2 m-1}\right)^{\frac{2 m}{B_{2 m}}}}{\left(D_{4 m-1}\right)^{\frac{8 m}{B_{4 m}}}}\right) .
$$

Similarly, by way of

$$
\frac{2}{p_{n}^{2 m}-p_{n}^{-2 m}}=\frac{1}{p_{n}^{2 m}-1}+\frac{1}{p_{n}^{2 m}+1} .
$$

we obtain the following identity.

Corollary 3.2 We have

$$
\prod_{n=1}^{\infty} p_{n}^{1 /\left(p_{n}^{2 m}-p_{n}^{-2 m}\right)}=\left(\frac{\left(D_{2 m-1}\right)^{\frac{1}{B_{2 m}}}}{\left(D_{4 m-1}\right)^{\frac{2}{B_{4 m}}}}\right)^{2 m} .
$$

It is simple to find further identities of the same type, although the reliance on fractions of the form $\left(p_{n}^{2 m}-1\right)^{-1}$ restricts the polynomials in the exponent of $p_{n}$.

Consider next the algebraic relation

$$
\sum_{m=1}^{i} \frac{A_{m}}{p_{n}^{2^{m}}-1}=\frac{\sum_{m=1}^{i} A_{m} \prod_{j=1}^{i-m}\left(p_{n}^{2^{i-j}}+1\right)}{p_{n}^{2^{i}}-1} .
$$

In the right-hand side of (3.1), we have factored the largest denominator, namely $p_{n}^{2^{i}}-1$, and then grouped terms. To illustrate how one might use this relation, we take $i=3, A_{1}=1,1+A_{2}+A_{3}=0$, and set $A_{2}=y$, to obtain

$$
\sum_{m=1}^{3} \frac{A_{m}}{p_{n}^{2^{m}}-1}=\frac{p_{n}^{2}\left(p_{n}^{4}+(1+y) p_{n}^{2}+1\right)}{p_{n}^{8}-1} .
$$

Employing this relation, Theorem 2.1 gives the following. 
Corollary 3.3 We have

$$
\prod_{n=1}^{\infty} p_{n}^{\frac{p_{n}^{2}\left(p_{n}^{4}+(1+y) p_{n}^{2}+1\right)}{p_{n}^{8}-1}}=D_{1}^{12} D_{7}^{240}\left(\frac{D_{7}^{240}}{D_{3}^{120}}\right)^{-y}
$$

Similarly, if we instead consider sums of the form

$$
\frac{1}{p_{n}^{2}-1}+\sum_{m} \frac{A_{m}}{p_{n}^{2 i m}-1}
$$

with integer $i$, we can obtain product relations of the following type.

Corollary 3.4 We have

$$
\prod_{n=1}^{\infty} p_{n}^{\frac{\left(p_{n}^{2}-y\right)\left(p_{n}^{2}+y+1\right)}{p_{n}^{6}-1}}=\frac{D_{1}^{12}}{D_{5}^{252}}\left(\frac{2 \pi e^{\gamma}}{D_{5}^{252}}\right)^{y+y^{2}} .
$$

While above we have considered finite sums of fractions of the type $\frac{1}{p_{n}^{2 m}-1}$, given a relation of the form

$$
\sum_{m=1}^{\infty} \frac{a_{m}}{p_{n}^{2 m}-1}=: f(n),
$$

one can convert further products over primes into products over the BenderskyAdamchik constants $D_{m}$. We shall explore this in the next section.

\section{Infinite products involving powers of Bendersky-Adamchik constants}

It is known (see the final proposition in [2]) that the Bendersky-Adamchik constants $D_{m}$ are related to the harmonic numbers $H_{n}$ and the Bernoulli numbers $B_{n}$ through

$$
\log D_{m}=\frac{B_{m+1} H_{m}}{m+1}-\zeta^{\prime}(-m) .
$$

We can deduce the behaviour of the $D_{2 m}$ from this formula. For all $m \geq 1, B_{2 m+1}=0$. For even index values, note

$$
\zeta^{\prime}(-2 m)=\frac{(-1)^{m} \zeta(2 m+1)(2 m) !}{2(2 \pi)^{2 m}},
$$

which follows from differentiation of Riemann's functional equation for the zeta function (see [20]), then evaluated at negative even integers. Interestingly, combining (4.2) with (4.1), it can be noted that the $D_{2 m}$ can be directly related to the odd values of the zeta function [7]

$$
D_{2 m}=\exp \left(\frac{(-1)^{m+1} \zeta(2 m+1)(2 m) !}{2(2 \pi)^{2 m}}\right) .
$$


In keeping with our aim of establishing infinite products related to the BenderskyAdamchik constants, we note that as (4.3) gives $D_{2 m}$ as an exponential, then evaluating products of $D_{2 m}$ for $m=1,2,3$ reduces to putting certain series into a closed-form. The simplest example is the product

$$
\prod_{m=1}^{\infty} D_{2 m}=\exp \left(\sum_{m=1}^{\infty} \frac{(-1)^{m+1} \zeta(2 m+1)(2 m) !}{2(2 \pi)^{2 m}}\right) \approx 1.0256
$$

where we have approximated the infinite series value numerically since it does not have a closed-form expression. To find other related products, we shall raise $D_{2 m}$ to a power sufficient to ensure that the product does converge. For example, one may show the following.

Corollary 4.1 We have

$$
\prod_{m=1}^{\infty} D_{2 m}^{\frac{1}{\xi(2 m+1)(2 m) !}}=\exp \left(\frac{1}{2} \sum_{m=1}^{\infty} \frac{(-1)^{m+1}}{(2 \pi)^{2 m}}\right)=\exp \left(\frac{1}{2} \frac{1}{4 \pi^{2}+1}\right)
$$

and

$$
\prod_{m=1}^{\infty} D_{2 m}^{\frac{(-1)^{m+1}}{\zeta(2 m+1)(2 m) !}}=\exp \left(\frac{1}{2} \sum_{m=1}^{\infty} \frac{1}{(2 \pi)^{2 m}}\right)=\exp \left(\frac{1}{2} \frac{1}{4 \pi^{2}-1}\right)
$$

The factor of $\zeta(2 m+1)$ in the exponent of the $D_{2 m}$ can be removed through appropriate exponentiation, giving a modified product as follows.

\section{Corollary 4.2 We have}

$$
\prod_{m=1}^{\infty} D_{2 m}^{\frac{(-1)^{m+1} \pi^{2 m}}{(2 m) !}}=\exp \left(\frac{1}{2} \sum_{m=1}^{\infty} \frac{\zeta(2 m+1)}{4^{m}}\right)
$$

Changing the order of summation (which we justify in the next section), one obtains

$$
\begin{aligned}
\sum_{m=1}^{\infty} \frac{\zeta(2 m+1)}{4^{m}} & =\sum_{m=1}^{\infty} \sum_{n=1}^{\infty} \frac{1}{n^{2 m+1} 4^{m}} \\
& =\sum_{n=1}^{\infty} \frac{1}{n}\left(\sum_{m=1}^{\infty} \frac{1}{\left(4 n^{2}\right)^{m}}\right) \\
& =\sum_{n=1}^{\infty} \frac{1}{n}\left(\frac{1}{4 n^{2}-1}\right) \\
& =\log (4)-1
\end{aligned}
$$

From here we have an elegant formula. 
Corollary 4.3 We have

$$
\prod_{m=1}^{\infty} D_{2 m}^{\frac{(-1)^{m+1} \pi^{2 m}}{(2 m) !}}=\frac{2}{\sqrt{e}} .
$$

A change in the order of summation can be applied to evaluate other series involving zeta functions. The partial fraction expansion of the cotangent function was first reported by Euler [8] and is given by

$$
\sum_{m=1}^{\infty} \frac{1}{m^{2}-z^{2}}=\frac{1-\pi z \cot (\pi z)}{2 z^{2}}
$$

which is valid for complex $z \notin \mathbb{Z}$. Using the identity (4.4), we find the following.

Corollary 4.4 We have

$$
\prod_{m=1}^{\infty} D_{2 m}^{\frac{(-1)^{m+1} \zeta(2 m)}{\zeta(2 m+1)(2 m) !}}=\exp \left(\frac{1}{4}\left(1-\frac{1}{2} \cot \left(\frac{1}{2}\right)\right)\right)
$$

and

$$
\prod_{m=1}^{\infty} D_{2 m}^{\frac{\zeta(2 m)}{\zeta(2 m+1)(2 m) !}}=\exp \left(\frac{1}{4}\left(1+\frac{1}{2} \operatorname{coth}\left(\frac{1}{2}\right)\right)\right)
$$

\section{Infinite double products}

We now extend our approach to the study of certain infinite double product, such as are studied in $[19,23]$. The following results will be useful.

Proposition 5.1 Suppose $x>1$, and that $a_{m}$ is a sequence such that

$$
\sum_{n=1}^{\infty} \sum_{m=1}^{\infty} \frac{\left|a_{m}\right| \log n}{n^{m x}}<\infty
$$

Then

$$
\sum_{m=1}^{\infty} \sum_{n=1}^{\infty} \frac{a_{m} \log n}{n^{m x}}=\sum_{n=1}^{\infty} \sum_{m=1}^{\infty} \frac{a_{m} \log n}{n^{m x}} .
$$

Proof This follows from Tonelli's Theorem for sequences [15], due to the absolute convergence of the double sum.

The above proposition has the following corollary, which we shall use.

Corollary 5.1 Suppose that $a_{m}$ is a sequence such that

$$
\prod_{n=1}^{\infty} n^{\sum_{m=1}^{\infty} \frac{\left|a_{m}\right|}{n^{m x}}}<\infty
$$


Then

$$
\prod_{m=1}^{\infty} \prod_{n=1}^{\infty} n^{a_{m} n^{-m x}}=\prod_{n=1}^{\infty} n^{\sum_{m=1}^{\infty} a_{m} n^{-m x}} .
$$

Proof After exponentiation of the result in Proposition 5.1, the corollary follows by noting

$$
\prod_{m=1}^{\infty} n^{\frac{a_{m}}{n^{2 m}}}=n^{\sum_{m=1}^{\infty} \frac{a_{m}}{n^{2 m}}}
$$

We now have the tools to show that the double product over terms of the form $n^{1 / n^{2 m}}$ is in fact convergent. Taking $a_{m}=1$, then for $n>1$,

$$
\sum_{m=1}^{\infty} \frac{a_{m}}{n^{2 m}}=\sum_{m=1}^{\infty} \frac{1}{n^{2 m}}=\frac{1}{n^{2}-1} .
$$

As $\left|a_{m}\right|=a_{m}$ here, and noting that for $n>1$ we have $\frac{1}{n^{2}-1} \leq \frac{1}{n^{3 / 2}}$, we see that

$$
1 \leq \prod_{n=2}^{\infty} n^{1 /\left(n^{2}-1\right)} \leq \prod_{n=2} n^{1 / n^{3 / 2}}<\infty .
$$

This allows us to apply Corollary 5.1. We now see that the double product over terms of the form $n^{1 / n^{2 m}}$ does indeed converge, as the corollary shows:

$$
\begin{aligned}
\prod_{m=1}^{\infty}\left(\frac{\left(D_{2 m-1}\right)^{\frac{2 m}{B_{2 m}}}}{2 \pi e^{\gamma}}\right)^{\zeta(2 m)} & =\prod_{m=1}^{\infty} \prod_{n=1}^{\infty} n^{1 / n^{2 m}} \\
& =\prod_{m=1}^{\infty} \prod_{n=2}^{\infty} n^{1 / n^{2 m}} \\
& =\prod_{n=2}^{\infty} n^{1 /\left(n^{2}-1\right)}<\infty .
\end{aligned}
$$

By defining the $a_{m}$ to be non-zero only at the primes, we can use Corollary 5.1 to evaluate infinite double products over the primes. For example, by taking $x=2 m$, we can use Theorem 2.1 and (5.1) to obtain the following closed-form double product.

Corollary 5.2 We have

$$
\prod_{k=1}^{\infty} \prod_{n=1}^{\infty} p_{n}^{1 / p_{n}^{2 k m}}=\frac{\left(D_{2 m-1}\right)^{\frac{2 m}{B_{2 m}}}}{2 \pi e^{\gamma}}
$$


Next, note that the equation

$$
\sum_{n=1}^{\infty} \frac{1}{p_{n}^{x}}=\sum_{k=1}^{\infty} \frac{\mu(k)}{k} \log (\zeta(k x))
$$

was shown to hold, for $x>1$, in [9]. Making use of our approach, we obtain another infinite double product identity.

Corollary 5.3 We have

$$
\prod_{k=1}^{\infty} \prod_{n=1}^{\infty} p_{n}^{\frac{\mu(k)}{p_{n}^{2 k m}-1}}=\prod_{n=1}^{\infty} p_{n}^{1 / p_{n}^{2 m}}=\prod_{k=1}^{\infty}\left(\frac{\left(D_{2 k m-1}\right)^{\frac{2 k m}{B_{2 k m}}}}{2 \pi e^{\gamma}}\right)^{\mu(k)}
$$

Recall from [17] that

$$
\sum_{p_{n} \leq x} \frac{\log p_{n}}{p_{n}}=\log x+O(1)
$$

The constant term in the asymptotic expansion is given by [17]

$$
C=\lim _{x \rightarrow \infty}\left(\log x-\sum_{p_{n} \leq x} \frac{\log p_{n}}{p_{n}}\right) \gamma+\log \left(\prod_{k=2}^{\infty} \prod_{n=1}^{\infty} p_{n}^{1 / p_{n}^{k}}\right)
$$

Making use of the absolute convergence of the double product in (5.2), and taking $m=1$ in (5.2), we have

$$
\frac{e^{C}}{e^{\gamma}}=\left(\prod_{k=1}^{\infty} \prod_{n=1}^{\infty} p_{n}^{1 / p_{n}^{2 k}}\right)\left(\prod_{k=1}^{\infty} \prod_{n=1}^{\infty} p_{n}^{1 / p_{n}^{2 k+1}}\right)=\frac{D_{1}^{12}}{2 \pi e^{\gamma}} \prod_{k=1}^{\infty} \prod_{n=1}^{\infty} p_{n}^{1 / p_{n}^{2 k+1}}
$$

Rearranging, we find an elegant identity, which is a double product generalization of products of Glaisher type.

Corollary 5.4 We have

$$
\prod_{k=1}^{\infty} \prod_{n=1}^{\infty} p_{n}^{1 / p_{n}^{2 k+1}}=\frac{2 \pi e^{C}}{D_{1}^{12}}
$$

Acknowledgements The authors appreciate reviewer comments which have greatly improved the presentation of the results.

Open Access This article is distributed under the terms of the Creative Commons Attribution 4.0 International License (http://creativecommons.org/licenses/by/4.0/), which permits unrestricted use, distribution, and reproduction in any medium, provided you give appropriate credit to the original author(s) and the source, provide a link to the Creative Commons license, and indicate if changes were made. 


\section{References}

1. Abramowitz, M., Stegun, I.A. (eds.): The Möbius function, section 24.3.1. In: Handbook of Mathematical Functions with Formulas, Graphs, and Mathematical Tables. Dover, New York (1972)

2. Adamchik, V.S.: Polygamma functions of negative order. J. Comput. Appl. Math. 100, 191-199 (1998)

3. Adamchik, V.S.: The multiple gamma function and its application to computation of series. Ramanujan J. 9(3), 271-288 (2005)

4. Bendersky, L.: Sur la fonction gamma généralisée. Acta Math. 61, 263-322 (1933)

5. Choi, J.: A set of mathematical constants arising naturally in the theory of the multiple gamma functions. Abstr. Appl. Anal. 2012 (2012) (Article ID 121795)

6. Choi, J., Srivastava, H.: Certain classes of series involving the zeta function. J. Math. Anal. Appl. 231, 91-117 (1999)

7. Choi, J., Srivastava, H.M., Adamchik, V.S.: Multiple gamma and related functions. Appl. Math. Comput. 134(2), 515-533 (2003)

8. Euler, L.: Introductio in Analysin Infinitorum. MM Bousquet, Lausanne (1748)

9. Glaisher, J.W.L.: On the sums of inverse powers of the prime numbers. Q. J. Math. 25, 347-362 (1891)

10. Glaisher, J.W.L.: On certain numerical products in which the exponents depend on the numbers. Messenger Math. 23, 145-175 (1893)

11. Glaisher, J.W.L.: On the constant which occurs in the formula for $1^{1} \cdot 2^{2} \cdot 3^{3} \ldots n^{n}$. Messenger Math. 24, 1-16 (1894)

12. Gould, H.W., Shonhiwa, T.: A catalog of interesting Dirichlet series. Mo. J. Math. Sci. 20, 2-18 (2008)

13. Guillera, J., Sondow, J.: Double integrals and infinite products for some classical constants via analytic continuations of Lerch's transcendent. Ramanujan J. 16(3), 247-270 (2008)

14. Heath-Brown, D.R.: The divisor function at consecutive integers. Mathematika 31, 141-149 (1984)

15. Junghenn, H.D.: A Course in Real Analysis. Chapman and Hall/CRC, Boca Raton (2015)

16. Lehman, R.S.: On Liouville's function. Math. Comput. 14, 311-320 (1960)

17. Mertens, F.: Ein Beitrag zur analytischen Zahlentheorie. J. Reine Angew. Math. 78, 46-62 (1874)

18. Milgram, M.S.: Integral and series representations of Riemann's zeta function, Dirichlet's eta function and a medley of related results. J. Math.2013 (2013) (Article ID 181724)

19. Mittag-Leffler, G.: A method of deriving the infinite double products in the theory of elliptic functions from the multiplication theorems. Ann. Math. 27, 187-194 (1926)

20. Riemann, B.: Ueber die Anzahl der Primzahlen unter einer gegebenen Grosse. Ges. Math. Werke Wiss. Nachlaß 2, 145-155 (1859)

21. Rota, G.-C.: On the foundations of combinatorial theory I. Theory of Möbius functions. Z. Wahrscheinlichkeitstheorie Verwandte Geb. 2, 340-368 (1964)

22. Titchmarsh, E.C., Heath-Brown, D.R.: The Theory of the Riemann zeta-Function. Oxford University Press, Oxford (1986)

23. Van Gorder, R.A.: Infinite multiple products over the primes of type $\prod \prod\left(1-p_{n}^{-p_{m}}\right)^{-1}$ and generalizations. Int. J. Contemp. Math. Sci. 5, 1499-1504 (2010)

24. Van Gorder, R.A.: Glaisher-type products over the primes. Int. J. Number Theory 8(2), 543-550 (2012)

25. Van Malderen, R.: Riemann's zeta function: the alternating xi-function Xia(s). arXiv:1610.04344 (2016)

26. Voros, A.: Spectral functions, special functions and the Selberg zeta function. Commun. Math. Phys. 110(3), 439-465 (1987) 